\title{
IDENTIFICATION VISUELLE DES GESTES DE PROTRUSION ET DE RETRACTION DES LËVRES AU COURS DES PAUSES ACOUSTIQUES : Les performances de sujets français et grees
}

\author{
M.A. CATHIARD, A. CIROT-TSEVA* et M.T. LALLOUACHE* \\ Laboratoire de Psychologie Expérimentale, URA CNRS $N^{\circ} 665$, Université Pierre Mendès-France, \\ $B P .47 X, F-38040$ Grenoble cedex, France \\ *Institut de la Communication Parlée, URA CNRS $N^{\circ} 368$, INPG-Université Stendhal, BP. $25 X$, \\ F-38040 Grenoble cedex, France
}

\begin{abstract}
Since coarticulation is one of the basic phenomena in speech production, the issue that speech perception could take advantage of it, is of course a very fundamental one. The main questions we address in this paper are, more specifically : (i) Could speech perception benefit from anticipatory - and perseverative - coarticulation when it is clearly visible, but inaudible, as in the case of vowel rounding (protrusion/retraction) along acoustic silent pauses, which occur naturally in discourse? (ii) Could speakers of a language like Greek, who do not have linguistically significant rounding contrasts, as in French, take the same visual advantage of coarticulation? Our results show that: (i) Rounding anticipation can be identified reliably (95\% correct), only by eye, up to 120 milliseconds before any perceivable sound; but this identification perseverate no more than $40-60 \mathrm{~ms}$ after sound utterance. (ii) The comparison between French and Greek subjects did not reveal significant differences in their identification behaviour, neither for anticipatory, nor perseverative coarticulation. Wether these results argue for a universal lipreading skill, remains of course an open quest.
\end{abstract}

\section{1-INTRODUCTION}

«In face-to-face communication speech can be "seen" before it is heard [s.p.n.], visual cues from lip movements may exist in some cases hundreds of milliseconds before the corresponding auditory stimulus». Cette affirmation récente d'Aulanko et Sams [1], qui présentent comme un fait acquis que la parole puisse être perçue visucllement, avant même que soit émis le signal acoustique correspondant, n’a pas été, à ce jour, documentée expérimentalement dans les publications consacrées à cette modalité ([2]; [3]), longtemps considérée comme secondaire. Seul [4] mentionne brièvement, dans ce sens, une expérience (non publiée, comm. pers.), qui révèle, par l'étude des temps de réaction, que des syllabes CV peuvent être identifiées à partir de la vision du mouvement des lèvres, avant d'être auditivement perçues. Par ailleurs, plus récemment, [5] ont pu montrer, pour des mouvements produits en cours d'émission sonore, que l'anticipation du geste d'arrondissement perçue visuellement n'était identifiée auditivement qu'environ $80 \mathrm{~ms}$ plus tard.

Les recherches que nous poursuivons [6] ont pour but d'évaluer précisẻment l'identification visuelle du trait d'arrondissement avant (et après) toute émission acoustique. Il s'agit en fait de montrer que les sujets parlants que nous sommes ont la capacité de tirer parti de l'anticipation et de la persévération des gestes linguistiques sur les lèvres. Ainsi, sachant que la disponibilité articulatoire de l'information de protrusion a pu déjà être mise en évidence à travers un ensemble de consonnes précédant une voyelle arrondie comme $[y]$, dans des séquences [i C...C y] (cf, pour le français, les travaux de [7] et plus généralement ceux de [8]), nous montrerons qu'elle est disponible aussi pendant les pauses acoustiques. Parallèlement, nous ferons appel à un phénomène de persévération peu connu : celui qui se produit dans la transition inverse, de la voyelle [y] vers la voyelle [i], à travers la pause, pour le geste de rétraction.

Sur ces deux phénomènes de coarticulation, nous examinerons plus particulièrement trois questions : (i) L'anticipation/persévération articulatoire naturclle d'un son, par rapport à son émission acoustique, peut-elle être récupérée seulement par la vision? (ii) Les gestes de protrusion et de rétraction sont-ils articulatoirement et perceptivement symétriques? (iii) Enfin, y a-t-il un déplacement des fronlières perceptives visuelles pour des sujets qui ne possèdent pas, comme en français, d'opposition phonologique reposant essentiellement sur l'arrondissement ([i] vs. [y]), ce qui est le cas de sujets grecs?

\section{2- MÉTHODE}

2.1. Corpus

Nous avons utilisé quatre trajectoires [V1\#V2] avec (V1,V2 = [i] ou [y]; \# étant la pause) qui étaient produites dans des phrases porteuses du type: «Tu dis: UHI ise?» et «T'as lu: IHU use?», où UHI, IHI, IHU et UHU sont, par convention, des prénoms d'indiens; «ise» et «use» étant en fonction de verbe. Les phrases [ $t$ y d i \# i i i: $z$ ] et [t a 1 y \# y y y: $z$ ] réalisent des transitions contrôle (faute de place, nous ne les examinerons pas ci-dessous). Avec dix répétitions, nous obtenons 40 phrases qui ont été enregistrées, en ordre aléatoire, dans deux conditions (deux prises de vues): une première fois en demandant au locuteur de marquer une pause courte [\#], puis une seconde fois, avec une pause plus longue [\#:]. 


\subsection{Enregistrement}

Un locuteur masculin français a été filmé, de face et de profil, à 50 images/seconde (son synchrone), dans une chambre sourde anéchoïque. Le locuteur, assis, la tête maintenue par un casque solidaire de son siège, est porteur d'une paire de lunettes noires qui lui protègent les ycux d'un éclairage puissant (1000 Watts halogènes). Sur la branche droite de ses lunettes est fixée une réglette qui servira de référence pour les mesures de profil. Les lèvres du locuteur sont maquillées en bleu : un Chroma-key est connecté à la sortie de la caméra de face pour transformer, en temps réel, la couleur bleue en noir saturé, dans le but de réaliser une détection maximale des contours externe et interne des lèvres (en levant toute ambiguïté entre le niveau de gris des lèvres et d'éventuelles ombres à l'intérieur de la cavité buccale).

2.3. Sélection des stimuli visuels

\subsubsection{Mesures acoustiques}

Après mesure de la durée des pauses intervocaliques, nous avons retenu deux réalisations, pour chacune des quatre transitions, l'une représentative de la durée moyenne des pauses courtes (\# $=160 \mathrm{~ms}$ ) et l'autre représentative des pauses longues (\#: $=460 \mathrm{~ms}$ ).

2.3.2. Étude articulatoire

Les images ( $512 \times 512$ pixels, sur 256 niveaux de gris) des signaux vidéo correspondant aux huit phrases retenues ont été soumises à un traitement d'images ([9]), ce qui nous a ainsi permis d'obtenir huit mesures frontales et latérales caractéristiques de la fente labiale et des protrusions des deux lèvres. L'examen des évolutions temporelles de la protrusion de la lèvre supérieure (P1) et de l'aire (S) - ainsi que de leurs dérivées obtenues après lissage par fonctions splines cubiques montre, pour la transition [ $\mathrm{i}$ \# y] (Fig.1), une anticipation importante du geste d'arrondissement des lèvres. Après une première rampe plutôt lente ([8]), les mouvements de protrusion et de constriction [diminution de l'aire] démarrent - d'après leurs pics d'accélération ([10]) - respectivement, à 160 et $120 \mathrm{~ms}$ (pour la petite pause) et à 240 et $200 \mathrm{~ms}$ (pour la grande), avant le début acoustique du [y]. Par contraste, la transition [y \# i] (Fig. 2) nous offre, sur le plan articulatoire, un décalage temporel des deux paramètres $\mathrm{P} 1$ et $\mathrm{S}$ de même amplitude, mais inverse, puisque le mouvement de rétraction démarre $40 \mathrm{~ms}$ en avance sur le relâchement de la constriction [augmentation de l'aire]. Ce décalage reste, de plus, relativement constant sur la partie la plus rapide du geste (par comparaison, dans la transition [i \# y], P1 et $S$ convergent plus vite, tout particulièrement dans la grande pause). Si nous estimons, par ailleurs, l'extension de la persévération de ces composantes $\mathrm{P} 1$ et $S$ par leurs pics de décélération, nous obtenons, respectivement, des valeurs de $10 \mathrm{ct} 100 \mathrm{~ms}$ (pour la petite pause) et de 80 et $140 \mathrm{~ms}$ (pour la grande), à partir de la fin acoustique du [y]. En résumé, on a ainsi, quelle que soit la durée de la pause, une anticipation plus précoce de l'aire par tapport à la protrusion dans $[i \rightarrow y]$ et une persévération plus tardive de cette même aire dans [y $\rightarrow \mathrm{i}$ ]. Mais, indépendamment de ce décalage inverse dans le timing des composantes du geste d'arrondissement, le phénomène majeur reste que, toutes choses égales par ailleurs, son anticipation est nettement plus longue que sa persévération.

2.4. Procédure du test de perception

Nous avons testé, pour les transitions à petite pause, 13 images (dont 9 pendant le silence de la pause); et pour les transitions à grande pause, 28 images (dont 24 de pause); avec 3 images avant le début et une après la fin de chaque pause. Ceci nous donne un total de 164 stimuli, qui ont été présentés en ordre aléatoire, avec un décalage de 5 images à chaque changement de sujet. En outre, 4 images étaient présentécs en début de test pour familiariser les sujets avec la tâche. La passation du test était individuelle. Il s'agissait d'un test didentification à choix forcé, le sujet devant décider pour chaque image si le locuteur prononçait un [i] ou un [y].

2.5. - Sujets

25 sujets de langue maternelle française et 24 sujets de langue maternelle grecque, sans déficit visuel ni auditif, ont participé au test d’identification. Aucun sujet n'avait de compétence particulière cn lecture labiale. Une bonne identification auditive du contraste [i] vs. [y] a été confirmée pour tous les sujets grecs (score moyen : 93.5\%).

\section{3 - RÉSULTATS.}

Nous avons calculé, pour chaque image, le pourcentage d'identification [y] obtenu par les sujets français, d'une part, et par les sujets grecs, d'autre part. Les courbes d'identification dressées pour chaque transition présentent une forme classique en sigmoïde (Fig. 1 et 2 ).

3.1. - Transition [i $\rightarrow$ y] (Fig.1)

La perception du trait d'arrondissement peut être anticipée visuellement de plusieurs dizaines de millisecondes, avant même que le moindre signal acoustique ait été émis, puisqu'aussi bien les sujets grecs que les sujets français identifient correctement l'information segmentale d'arrondissement ( $95 \%$ de réponses [y]), $60 \mathrm{~ms}$ avant le début acoustique du [y], dans la petite pause, et $120 \mathrm{~ms}$, dans la grande pause. Pour déterminer la frontière perceptive visuelle sur nos fonctions d'identification, nous avons utilisé l'analyse Probit [11] qui permet : (i) d'estimer pour chaque courbe, la position temporelle de sa frontière à $50 \%$ (sa moyenne) et sa pente, en utilisant toutes les données comprises entre les régions asymptotiques; (ii) de comparer les courbes sur leurs frontières, par un test de type Sudent, après avoir testé leur parallélisme. Les ajustements effectués montrent que la frontière se silue : pour la petite pause, à $90 \mathrm{~ms}$ avant le début acoustique du [y], pour les sujets français, et à $80 \mathrm{~ms}$, pour les sujets grecs; et pour la grande pause, à $180 \mathrm{~ms}$ et à $190 \mathrm{~ms}$, respectivement. Entre les deux conditions de pause, il y a une différence significative $(p<.01)$, pour les deux groupes de sujets : la position temporelle de la frontière dépend donc de l'extension de la pause, autrement dit, quand la pause triple, l'anticipation visuelle double. Par contre, la précision temporelle de ces mêmes frontières - estimée par leurs pentes - ne dépend pas de la longueur des pauses. En effet, 80 à $100 \mathrm{~ms}$ suffisent, dans les deux conditions, pour passer nettement de [i] à [y]. La comparaison des frontières visuelles des sujets français et grecs, pour chaque valeur de pause, n'indique pas de différences significatives. On remarquera que le démarrage des fonctions d'identification (leurs pies de dérivée seconde : images $n^{\circ} 16$ pour la grande pause; dans la petite pause, image $n^{\circ} 5$ pour les français et $n^{\circ} 6$ pour les grecs) se situe aux alentours des démarrages (pics d'accélération) des composantes $\mathrm{P} 1$ et $\mathrm{S}$. 

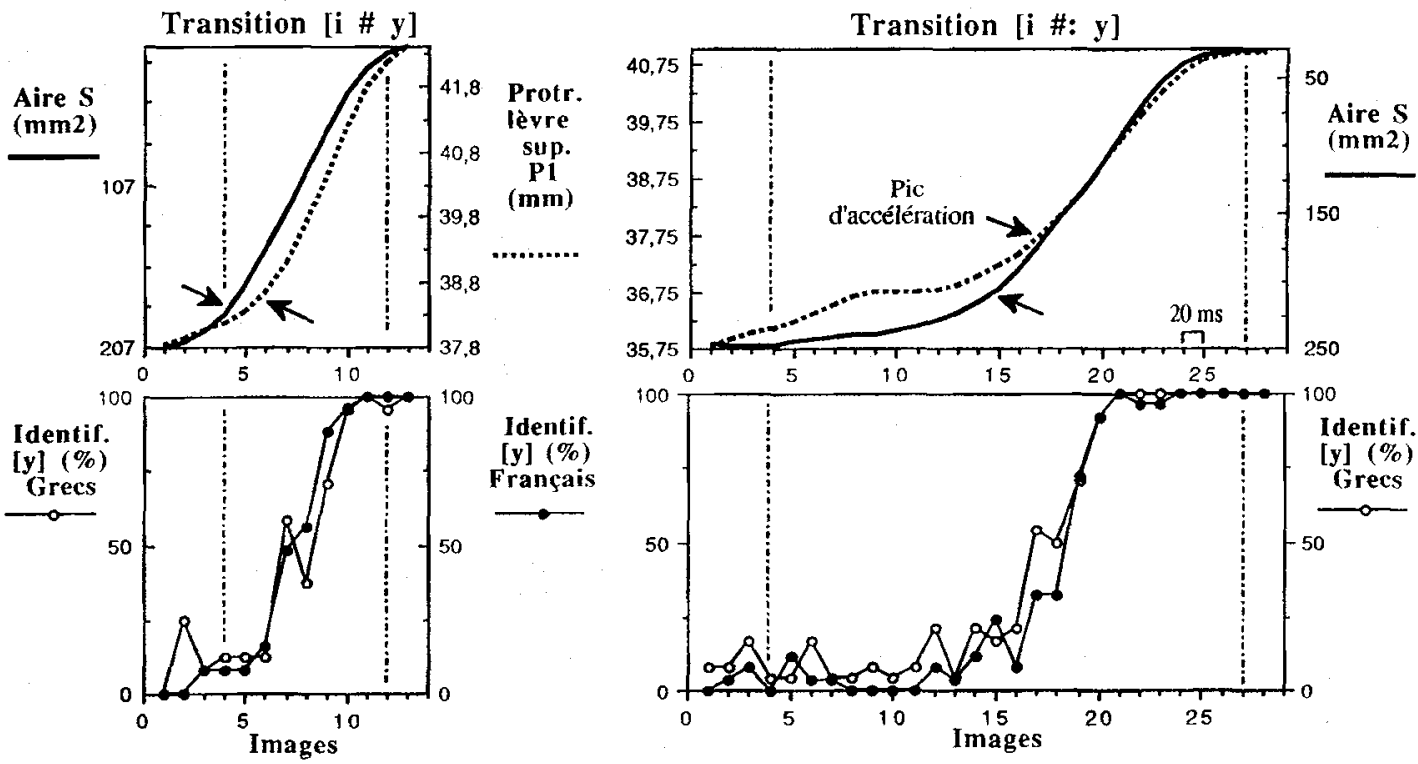

Figure 1. : Transitions [i $\rightarrow$ y] avec petite (\#) et grande pause (\#:) - En haut : Évolution de la protrusion de la lèvre supérieure (Pl) et de l'aire (S), après lissage par fonctions splines cubiques (les flèches indiquent les pies d'accélération).

- En bas : Fonctions d'identification [y] correspondantes pour 25 sujets français et 24 sujets grecs.

La verticale point-tiret de gauche indique la fin acoustique du li]; celle de droite, le début acoustique du [y].

Transition [y \# i]

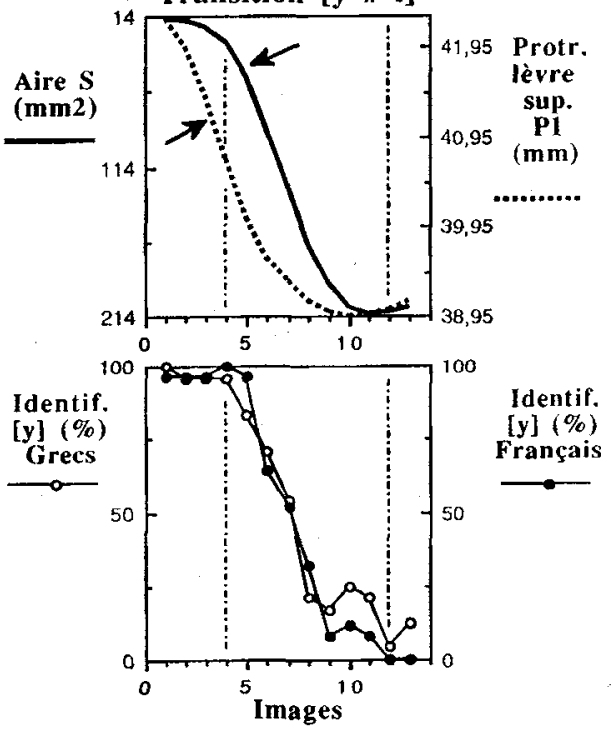

Transition [y \#: i]

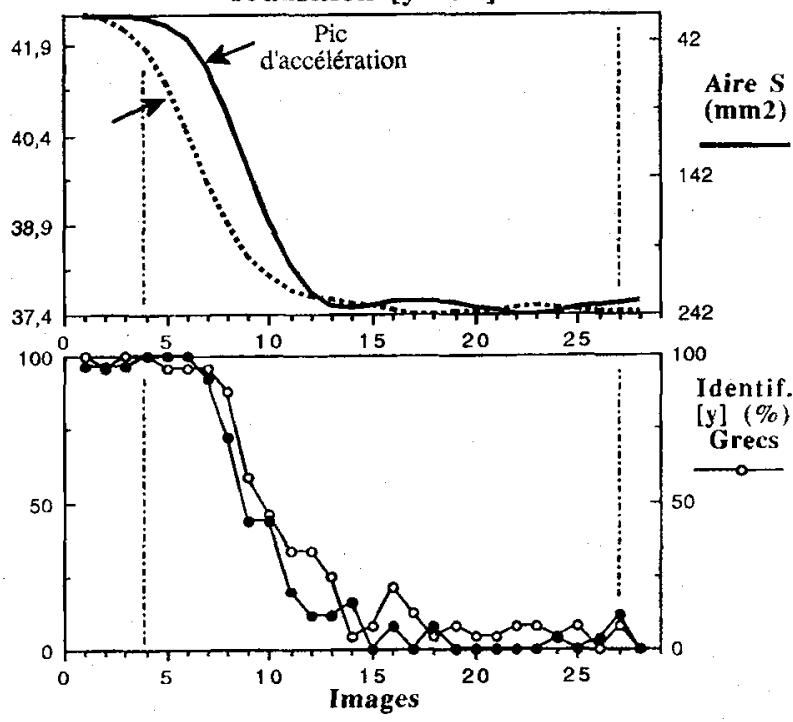

Figure 2. : Transitions $[y \rightarrow$ i] avec petite (\#) et grande pause (\#:)

- En haut : Évolution de la protrusion de la lèvre supérieure (P1) et de l'aire (S), après lissage par fonctions splines cubiques (les flèches indiquent les pics d'accélération).

- En bas : Fonctions didentification $|y|$ correspondantes pour 25 sujets français et 24 sujets grecs.

La verticale point-tiret de gauche indique la fin acoustiçue du $\{y\}$; celle de droite, le début acoustique du fi]. 


\section{2. - Transition [y $\rightarrow$ i] (Fig. 2)}

La perception visuelle de l'arrondissement, dans la transition de la voyelle [y] vers la voyelle [i], à travers la pause, peut persévérer plus ou moins après la fin de l'émission acoustique de la voyelle : à $95 \%$ de rêponses correctes [y], les sujets grecs identifient l'information segmentale visuelle d'arrondissement de 0 à $60 \mathrm{~ms}$, après le début de la pause; les sujets français de 20 à $40 \mathrm{~ms}$. La frontière visuelle des courbes estimée à $50 \%$ par Probit se situe : pour les sujets français et grecs, $60 \mathrm{~ms}$ après la fin acoustique du [y], dans la petite pause; dans la grande pause, $110 \mathrm{~ms}$ après, pour les français, et $120 \mathrm{~ms}$ après, pour les grecs; ces valeurs sont loin d'être négligeables, mais elles restent toutes inférieures aux valeurs d'anticipation correspondantes (cf. 3.1.). La différence, entre les deux conditions de pause, pour les deux groupes de sujets, s'avère significative $(\mathrm{p}<.01)$. Là aussi, comme pour l'anticipation - et même si les durées de persévération sont nettement plus faibles - lorsque la pause triple, la persévération visuelle double. La précision temporelle des frontières est identique à celle observée dans la transition [i $\rightarrow$ y]. Il $n^{\prime} y$ a, comme précédemment, aucune différence significative entre les résultats des sujets français et grecs. De même que pour la transition $[\mathrm{i} \rightarrow \mathrm{y}]$, on remarquera que le démarrage des fonctions d'identification (images $n^{\circ} 4$ pour la petite pause; dans la grande pause, image $n^{\circ} 6$ pour les français et $n^{\circ} 7$ pour les grecs) se situe, là aussi, aux alentours des démarrages des composantes $\mathrm{P1}$ et $\mathrm{S}$.

\section{4.- CONCLUSIONS}

Dans cette étude sur la perception visuelle de l'anticipation et đe la persévération du trait d'arrondissement en français, nous avons utilisé le contrôle de la durée des pauses acoustiques. Dans la perception de la parole, ces pauses - qui ont une fonction prosodique évidente - nous offrent un cas naturel où l'information segmentale ne peut pas être récupérée pendant le silence autrement que par le signal optique, en mettant ainsi à profit le fait que celui-ci n'est pas interrompu.

De fait, nous avons obtenu les résultats suivants :

(i) L'anticipation naturelle du trait d'arrondissement lui permet d'être identifié seulement par la vision, et ceci de manière sûre (à 95\%), jusqu'à $120 \mathrm{~ms}$ avant que le moindre signal acoustique ait été émis. La frontic̀re [i]/[y] (à 50\%) se situe, dans la grande pause, $185 \mathrm{~ms}$ (en moyenne) avant le son; $85 \mathrm{~ms}$ dans la petite. La perception de la persévération de cet arrondissement - dans la transition de la voyelle [y] vers la voyelle [i], à travers la pause - s'étend nettement moins loin : elle n'est clairement identifiable (à $95 \%$ ) que $40-60 \mathrm{~ms}$ au plus après la fin de lémission acoustique de la voyelle. La frontière [y]/[i], dans la grande pause, est à $115 \mathrm{~ms}$ de la fin du son vocalique; à $60 \mathrm{~ms}$ dans la petite. Dans les deux cas - en anticipation comme en persévération - la coarticulation perçue double lorsque la pause triple. Mais ces deux perceptions avant et après le son - ont un empan temporel très différent : cn moyenne l'anticipation est une fois et demie plus grande que la persévération.

(ii) L'étude articulatoire de nos stimuli montre effectivement que les deux phénomènes ne sont pas symétriques. Cela est vrai pour l'extension de l'anticipation qui est globalement bien plus grande que celle de la persévération. Mais cela est aussi vrai jusque dans les composantes de ces gestes : on a ainsi, quelle que soit la durée de la pause, une anticipation plus précoce de l'aire $(\mathrm{S})$ par rapport à la protrusion $(\mathrm{P} 1)$ dans $[\mathrm{i} \rightarrow \mathrm{y}]$ et une persévération plus tardive de cette même aire dans $[y \rightarrow$ i]. Dans les deux cas, on remarque que le démarrage des fonctions didentification se situe, aux alentours des démarrages de ces composantes P1 et S. Une expérimentation, en cours, avec présentation de séquences dynamiques, devrait nous permettre d'évaluer l'importance perceptive de ces événements d'accélération du mouvement.

(iii) La comparaison des identifications des sujets français et des sujets grecs n'a pas mis en évidence de différences significatives sur les frontières visuelles. Rappelons que nos sujets grecs ont fait montre d'une compétence plutôt bonne en identification auditive de [i] $v s$. [y] (alors que leurs productions [y] sont habitucllement biaisées vers [i]). D'autre part, ils peuvent avoir lu le choix proposé «U» comme [u]. Quoi qu'il en soit, il reste que, dans les deux cas ([y] ou [u]), ils ne détectent pas significativement moins bien que les français l'anticipation et la persévération du trait vocalique d'arrondissement. Resterait à expliquer cette égalité de compétence en lecture labialé, tout particulièrement en ce qui conceme la capacité de récupérer visuellement - avant même toute émission sonore - les grandes classes de voyelles universellement présentes dans les langues du monde, arrondies [u] et non-arrondies [i].

Remerciements à Willy Serniclaes et à Jean-Luc Schwartz pour nous avoir guidés dans l'analyse Probit.

\section{REFERENCES}

[1] Aulanko, R. \& Sams, M., in Proceedings of the XIIth International Congress of Phonetic Sciences, 19-24 Août 1991, Aix-en-Provence, 4 (1991) 38-41.

[2] Massaro, D.W. , "Speech perception by ear and eye: a paradigm for psychological inquiry", (1987), Laurence Erlbaum Associates, London.

[3] Cathiard M.-A., Bull. Inst. Phonétique de Grenoble, 17-18 (1988/89) 109-193.

[4] McGurk, H., in T. Myers, J. Laver \& J. Anderson (Eds.), The Cognitive Representation of Speech, (1981), Amsterdam, North-Holland, 336-338.

[5] Escudier P., Benoit, C. \& Lallouache M.-T., JASA, Suppl. 1 (1990) 87 S126.

[6] Cathiard M.-A., Tiberghien G., Cirot-Tseva A., M.-T. Lallouache \& Escudier P., in Proceedings of the XIIth International Congress of Phonetic Sciences, 19-24 Août 1991, Aix-en-Provence, 4 (1991) $50-53$.

[7] Benguérel, A.P. \& Cowan, H.A., Phonetica, 30 (1974) 41-55.

[8] Perkell J.S., in W.J. Hardcastle \& A. Marchal (Eds.), Speech production and speech modelling, (1990), Kluwer Academic Publishers, Dordrecht, Bosion, London.

[9] Lallouache, M.-T.,(1991), "Un poste 'visage-parole' couleur. Acquisition et traitement automatique des contours des lèvres", thèse de l'ENSER, Grenoble.

[10] Perkell \& Matthies, JASA, Suppl. 1 (1990) 87 S123.

[11] Finney, D.J., Probit analysis, (1971), Cambridge University Press. 